\title{
Radial pseudoaneurysm following diagnostic coronary angiography
}

\author{
Shankar Laudari ${ }^{1}$, Sachin Dhungel ${ }^{1}$, Laxman Dubey ${ }^{1}$, Guru Prasad ${ }^{2}$, R Bhattacharya ${ }^{2}$, S Subramanyam ${ }^{2}$ \\ DM Residents ${ }^{1}$, Professors ${ }^{2}$, Department of Cardiology, COMS-TH, Bharatpur, Nepal
}

\begin{abstract}
The radial artery access has gained popularity as a method of diagnostic coronary catheterization compared to femoral artery puncture in terms of vascular complications and early ambulation. However, very rare complication like radial artery pseudoaneurysm may occur following cardiac catheterization which may give rise to serious consequences. Here, we report a patient with radial pseudoaneurysm following diagnostic coronary angiography. Adequate and correct methodology of compression of radial artery following puncture for maintaining hemostasis is the key to prevention.
\end{abstract}

Key words: Coronary catheterization, Pseudoaneurysm, Radial artery

\section{INTRODUCTION}

Transradialarterial puncture for diagnostic coronary angiography and percutaneous coronary intervention (PCI)is gradually replacing femoral artery access in many cardiac centers. This is because of the significant reduction in the occurrence of accesssite complications as well as patient comfort and early ambulation observed while selecting transradial over transfemoral puncture.Clinically significant pseudoaneurysm occurs in $0.05 \%$ to $1.0 \%$ of diagnostic and up to $6 \%$ of interventional transfemoral procedures. ${ }^{1,2}$ Posttransradial catheterization pseudoaneurysm is rare, with an incidence $<0.1 \%$ reported in a large caseseries. ${ }^{3}$

\section{CASE REPORT}

62 years female with past history of diabetes and systemic hypertension had undergone diagnostic coronary angiography via radial access for evaluation

Correspondence: Shankar Laudari

E-mail: lshankar2@hotmail.com and management of exertional chest pain and shortness for breath. The patient was on aspirin, clopidogrel, atorvastatin, nitrate and metoprolol. Bleeding and coagulation profiles were normal before the procedure. During angiography, single right radial artery puncture was done and coronary ostium hooked with diagnostic 5FrenchTiger catheter. Coronary angiography was suggestive of double vessel disease with $99 \%$ stenosis of ramus intermedius and $90 \%$ stenosis of left circumflex vessel. There was no local bleeding during and after the procedure but she presented with a pulsatile swelling of $2 \times 2 \mathrm{~cm}$ over distal wrist on the fourth day of intervention as shown in Fig 1. Systolic bruit was audible. Allen's test was negative. Radial Colour Doppler(as shown in Fig 2a and 2b) revealed the presence of pseudoaneurysm arising from the main right radial artery with continuous bidirectional blood flow in the neck of the pseudoaneurysm. There was turbulent blood flow within the lesion and in the radial artery which was patent. Ulnar artery and palmar arch 
Journal of College of Medical Sciences-Nepal, 2014, Vol-10, No-3

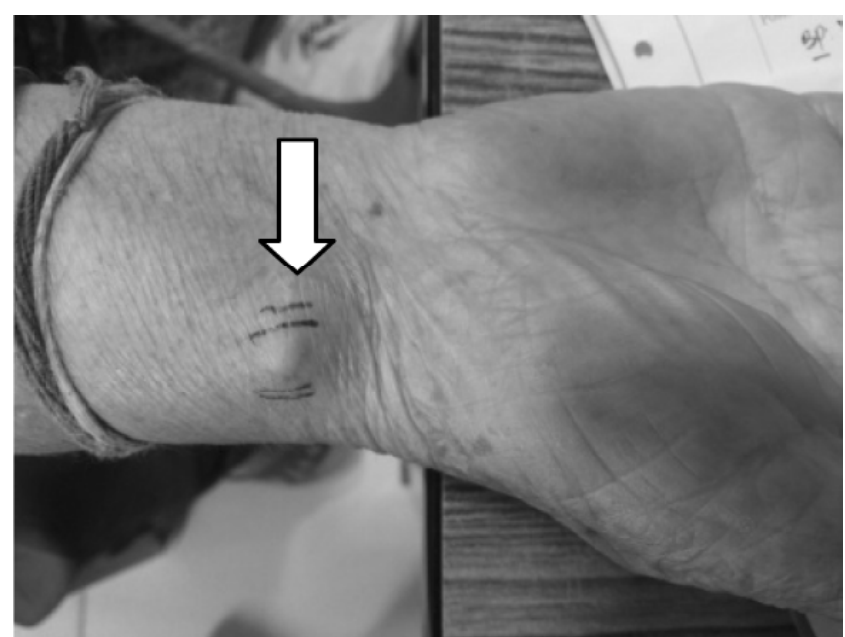

Fig 1:Pseudoaneurysmal swelling over right distal wrist



Fig 2a

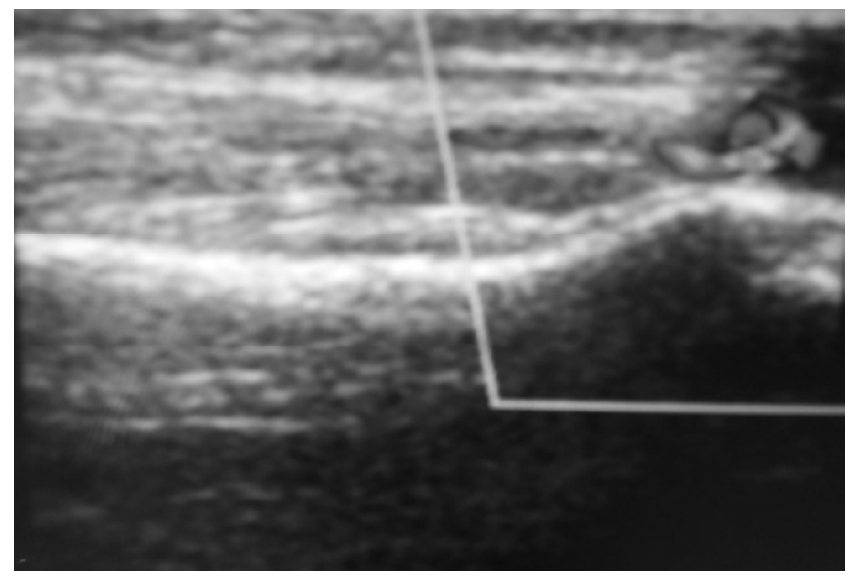

Fig 2b

Fig 2a and 2b: Doppler ultrasound of pseudoaneurysm (longitudinal view). Colour Doppler imaging showing flow of blood from lower end of right radial artery to hematoma cavity through a narrow neck.

\section{DISCUSSION}

The transradial access for cardiac catheterization has resulted in significant reduction of vascular complications. ${ }^{4}$ Complications of transradial catheterization include radial artery occlusion, nonocclusive injury, spasm, hand ischemia, nerve damage, bleeding and pseudoaneurysm formation. ${ }^{4}$

Factors predisposing to the development of radial artery pseudoaneurysm include multiple puncture attempts, ongoing systemic anticoagulation, inadequate hemostasis/postprocedure compression, vascular site infection and the use of larger catheter sheath sizes. ${ }^{5,6}$ Our patient had single radial artery puncture and received antiplatelet therapy before the procedure but not affected by access-site infection.

Postprocedure hemostatic compression devices are frequently used for the prevention of pseudoaneurysm formation. ${ }^{7}$ In our patient, manual compression was done for about 30 minutes. Potential problems may be incorrect and inadequate compression pressure. Attending nurses and doctors should be vigilant for proper clinical observation and assessment over radial puncture site following cardiac catheterization. Following clinical suspicion, confirmation of pseudoaneurysm with Colour Doppler Study and subsequent intervention is of extreme importance for preventing evolution of a hematoma and associated complications. $^{8}$

An individualized approach to management based on the severity of the pseudoaneurysm is recommended. ${ }^{4}$ For small defects, firm compression with a view to thrombose false aneurysm may suffice whereas larger aneurysms require surgical intervention. Where there is evidence of adequate collateral arterial flow to the hand, ligation and excision of the pseudoaneurysm and 
Journal of College of Medical Sciences-Nepal, 2014, Vol-10, No-3

radial artery may be permitted. ${ }^{9}$ Management using thrombin injection has also been reported but is unlikely to offer significant advantage over surgical intervention. ${ }^{8,9}$

\section{CONCLUSION}

Although vascular complications like pseudoaneurysm following radial artery puncture are very rare, they are significant and may result in serious catastrophes. Adequate and correct optimal postprocedure compression along with vigilant monitoring may minimize the risk of pseudoaneurysm formation.

\section{COMPETING INTERESTS}

The authors do not have competing interests in the publication of this article.

\section{REFERENCES}

1. Katzenschlager R, Ugurluoglu A, Ahmadi A. Incidence of pseudoaneurysm after diagnostic and therapeutic angiography. Radiology 1995;195:463-6.

2. Kresowik TF, Khoury MD, Miller BV. A prospective study of the incidence and natural history of femoral vascular complications after percutaneous coronary angioplasty. J VascSurg1991;13:328-36.

3. Sanmartin M, Cuevas D, Goicolea J, et al. Vascular complications associated with radial artery access for cardiac catheterization. Rev EspCardiol 2004;57:581-4.

4. Kanei Y, Kwan T, Nakra NC. Transradial cardiac catheterization: A review of access site complications. Catheter Cardiovasc Interven 2011;78:840-6.

5. Gilchrist IC. Laissez-faire hemostasis and transradial injuries. Catheter Cardiovasc Interv 2009;73:473-4.

6. Lefevre T, Morice MC, Bonan R. Coronary angiography using 4 or 6 French diagnostic catheters: A prospective, randomized study. J Invasive Cardiol2001;13:674-7.

7. Liou M, Tung F, Kanei Y, et al. Treatment of radial artery pseudoaneurysm using a novel compression device. J Invasive Cardiol2010;22:293-5.

8. Tahir H, Luke H, John Mc D. Radial artery pseudoaneurysm following coronary angiography in two octogenerians. ExpClinCardiol 2012;17: 260-62.

9. D'Achille A, Sebben RA, Davies RP. Percutaneous ultrasound-guided thrombin injection for coagulation of post-traumatic pseudoaneurysms. Australas Radiol 2001;45:218-21. 